\title{
Two novel mutations in the glycine decarboxylase gene in a boy with classic nonketotic hyperglycinemia: case report
}

\author{
Shu Liu, M.D. ${ }^{a}$, Zhiqing Wang, M.D. ${ }^{b}$, Jinqun Liang, B.S. ${ }^{a}$, Nuan Chen, B.S. ${ }^{a}$, Haimei OuYang, B.S. ${ }^{a}$, \\ Weihong Zeng, B.S. ${ }^{a}$, Liying Chen, B.S. ${ }^{a}$, Xunjie Xie, B.S. ${ }^{a}$ and Jianhui Jiang, Prof. M.D. ${ }^{a}$
}

\begin{abstract}
Nonketotic hyperglycinemia is an extremely rare autosomal recessively inherited glycine encephalopathy caused by a deficiency in the mitochondrial glycine cleavage system, which leads to severe clinical symptoms. Nonketotic hyperglycinemia is characterized by complex and diverse phenotypes, such as hypotonia, seizures, cognitive impairment, developmental delays and myoclonic jerks that may lead to apnea and even death. Here we report a 1-year-old boy with myoclonic seizures, hypotonia and coma; he had elevated plasma and cerebrospinal fluid glycine levels, and cerebrospinal fluid/plasma glycine ratio was 0.24 . Two novel heterozygous mutations confirm the diagnosis of nonketotic hyperglycinemia. One is a missense mutation c.2516A $>\mathrm{G}$ (p.Y839C) and the other one is a splicing mutation c. $2457+2 \mathrm{~T}>\mathrm{A}$ in the GLDC gene.

Key words: nonketotic hyperglycinemia, GLDC gene, novel mutations, glycine decarboxylase, encephalopathy.
\end{abstract}

http: / / dx.doi.org/10.5546/ aap.2017.eng.225

To cite: Liu S, Wang Z, Liang J, et al. Two novel mutations in the glycine decarboxylase gene in a boy with classic nonketotic hyperglycinemia: case report. Arch Argent Pediatr 2017;115(4):e225-e229.

\section{INTRODUCTION}

Nonketotic hyperglycinemia (NKH, OMIM 605899), also known as glycine encephalopathy, is a devastating autosomal recessive disorder of glycine metabolism. ${ }^{1,2}$ It can result in the accumulation of large amounts of glycine in body fluids and also can lead to severe neurologic dysfunction. As many inborn errors, a spectrum of phenotypic variation exists that result in the disorder. These variants, known as neonatal,

a. Children Inherited Metabolism and Endocrine Department, Guangdong Women and Children Hospital, Guangzhou, Guangdong 511400, P.R. China.

b. Guangdong Provincial Key Laboratory of Gastroenterology, Department of Gastroenterology, Nanfang Hospital, Southern Medical University, Guangzhou, China.

E-mail address:

Jianhui Jiang, M.D.: cimedgd@163.com

Funding: None.

Conflict of interest: None.

Received: 10-19-2016

Accepted: 3-28-2017 infantile, mild episodic, late onset and transitory, are characterized by heterogeneous phenotypes that include seizures, behavioral problems, cognitive deficits, and developmental delays. Most patients have the neonatal form, with the manifestations of glycine encephalopathy in the first few days of life with hypotonia, lethargy, intractable seizures. Some of them survive and will have intellectual disability in the future.,4

$\mathrm{NKH}$ is caused by a defect in the mitochondrial glycine cleavage system, ${ }^{3,5}$ which is diagnosed biochemically and molecularly. NKH is suspected in individuals with neurological symptoms accompanied by elevated glycine concentration in blood and cerebrospinal fluid (CSF). The increase of glycine concentration in CSF $(>80 \mu \mathrm{mol} / \mathrm{L})$ together with the increased glycine CSF/plasma level ratio (normally <0.02) strongly suggest the typical nonketotic hyperglycinemia. ${ }^{5}$ The final diagnosis for NKH should be determined by mutation detection of the glycine cleavage system genes. The glycine cleavage system is a mitochondrial multienzyme system consisting of four individual subunits, including three specific components: $P$ protein (glycine decarboxylase), $\mathrm{T}$ protein (aminomethyl transferase) and $\mathrm{H}$ protein (glycine cleavage system $\mathrm{H}$ protein), and one house-keeping enzyme: dihydrolipoamide dehydrogenase, which are respectively encoded by GLDC, AMT, GCSH and DLD genes. . $^{3,6,7}$ Mutations in the GLDC gene are responsible for the majority of the of NKH cases. ${ }^{3}$ For the first time, we report a 1-year-old boy with neonatal NKH who is heterozygous for two novel mutations in the GLDC gene.

\section{Case study}

Our patient is a 1-year-old boy with a history of hypotonia and frequent twitching of the limb who was born to non consanguineous parents. The boy was born in another hospital at 40 weeks of gestation to a 25-year-old, primiparous mother after an uneventful pregnancy with normal fetal movements (birth weight, $3150 \mathrm{~g}$ ). He was hospitalized in a neonatal intensive care unit because of poor feeding and decreased activity on the first day after his birth. He became 
lethargic, with generalized muscular hypotonia, poor feeding, absent deep tendon reflexes and developmental delay. From 3 months old, progressive myoclonic seizures were observed about 10 times a day.

Routine laboratory findings, including glycemia, serum ammonia, blood cells count, and blood coagulation function, were normal. Magnetic resonance imaging (MRI) demonstrated dysplasia of corpus callosum. The CSF study indicated a normal leukocyte amount, protein and glucose levels. The culturing and viral examination of CSF were found negative. CSF samples were analyzed using the PerkinElmer NeoBase non-derivatized MS / MS kit ${ }^{\mathrm{TM}}$ (3040-001Z), and procedure was performed as the standard protocol. AB Sciex API 3200 LC/MS/MS were used for analysis, and data management was done by the Chemoview ${ }^{\mathrm{TM}}$, PerkinElmer. Metabolic investigation showed the plasma glycine level increased to $947.8 \mu \mathrm{M} / \mathrm{L}$ (reference value, $115-600 \mu \mathrm{M} / \mathrm{L}$ ) and CSF glycine level reached $226.4 \mu \mathrm{M} / \mathrm{L}$ (reference value, 3-20 $\mu \mathrm{M} / \mathrm{L}$ ). The ratio of CSF to plasma glycine was 0.24 (normal $<0.02$ ). Thus, the analysis of amino acids in the CSF and plasma, along with the typical clinical manifestation, proved the diagnosis of nonketotic hyperglycinemia.

The diagnosis was confirmed by direct sequencing of all exons and intron-exon boundaries of GLDC, GCSH, and $A M T$, using DNA extracted from the peripheral lymphocytes. The boy carries two novel compound heterozygote mutations, which are c. $2457+2 \mathrm{~T}>\mathrm{A}$ and c.2516A $>G(Y 839 C)$ in exons 20 and 21 of $G L D C$ inherited from the father and mother respectively (Figure 1 B1-B4). So far among the existing research, these two mutations have not been reported previously.

Bioinformatic analysis of these two mutations were done by SIFT, Mutation Taster, Polyhen2, BDGP and ESE finder (Fig. $1 A, C-G$ ). The results were as follows: (1) c.2457+2T>A: Mutation Taster: disease causing, prob: 1, ESE finder: the mutation leads to the loss of the motif site, BDGP: the splice site predictions indicated that this site of wildtype formed a donor site of alternative splicing, with a score of 0.99 . (2) c.2516A $>$ G(Y839C): SIFT: damaging with a score of 0, Mutation Taster: disease causing, prob: 0.999999999819036, and Polyhen2: probably damaging with a score of 1.000 (the maximum value). Results of bioinformatic analysis strongly suggested that they were disease-causing mutations.
So far there is no effective treatment for NKH. The current treatment consists of reducing the glycine levels with benzoate and blocking the effect of excess glycine on the N-methyl-D-aspartate (NMDA) receptors with dextromethorphan ${ }^{8}$. In this case, oral administration of sodium benzoate at doses of $500 \mathrm{mg} / \mathrm{kg} / \mathrm{d}$, pyridoxine $300 \mathrm{mg} / \mathrm{d}$ and dextromethorphan $6 \mathrm{mg} / \mathrm{kg} / \mathrm{d}$ is used experimentally as a part of the treatment. Meanwhile, as a supplementary therapeutic method, dietary restriction of natural protein intake started, which was $3 \mathrm{~g} / \mathrm{kg} / \mathrm{d}$ at the time of diagnosis. To achieve targeted therapeutic effect, further dosage adjustments were based on actual blood glycine levels and patient's response. After one month of combined treatment, the muscular tone was improved slightly, and the frequency of seizure decreased to once or twice a day in first return visit, and the glycine levels of plasma drops to $752 \mu \mathrm{M} / \mathrm{L}$ (reference value, $115-600 \mu \mathrm{M} / \mathrm{L}$ ). According to this results, dosage adjustment of sodium benzoate at $600 \mathrm{mg} / \mathrm{kg} / \mathrm{d}$ and dextromethorphan at $12 \mathrm{mg} / \mathrm{kg} / \mathrm{d}$ started. His plasma glycine level normalized $(297 \mu \mathrm{mol} /$ L;reference range, $158-302 \mu \mathrm{mol} / \mathrm{L}$ ). Natural protein intake decreased to $2 \mathrm{~g} / \mathrm{kg} / \mathrm{d}$ after dosage increase of sodium benzoate. Before losing child follow-up, after half year's therapy, the glycine plasma levels fluctuated from 546 to $1132 \mu \mathrm{M} / \mathrm{L}$ (reference value, $115-600 \mu \mathrm{M} / \mathrm{L}$ ) because of poor treatment compliance, such as discontinuous drug therapy and poor protein-intake control. The last follow-up evaluation was at half a year after completion of the initial treatment (one year and seven months old), protein restricted diet and drug therapy improved the brisk deep tendon reflexes and muscular hypotonia, but it had no obvious effects on poor feeding and mental retardation.

\section{DISCUSSION}

The pathogenesis of $\mathrm{NKH}$ is relevant to accumulation of glycine in central nervous system as a result of glycine cleavage system deficiency. ${ }^{9}$ Symptoms can be explained by the neurotransmitter function of glycine. Glycine has an inhibitory function over specific receptors in the brainstem. The inhibitory function is enhanced when the level of glycine is higher, leading to respiratory insufficiency and lethargy. Glycine is also an obligatory coagonist with glutamate of the excitatory N-methyl-D-aspartate receptor (NMDAR) in cerebral cortex and cerebellum, 
FIGURE 1. Identification of the c.2457+2T>A and c.2516A>G(Y839C) mutations in exons 20 and 21 of GLDC gene
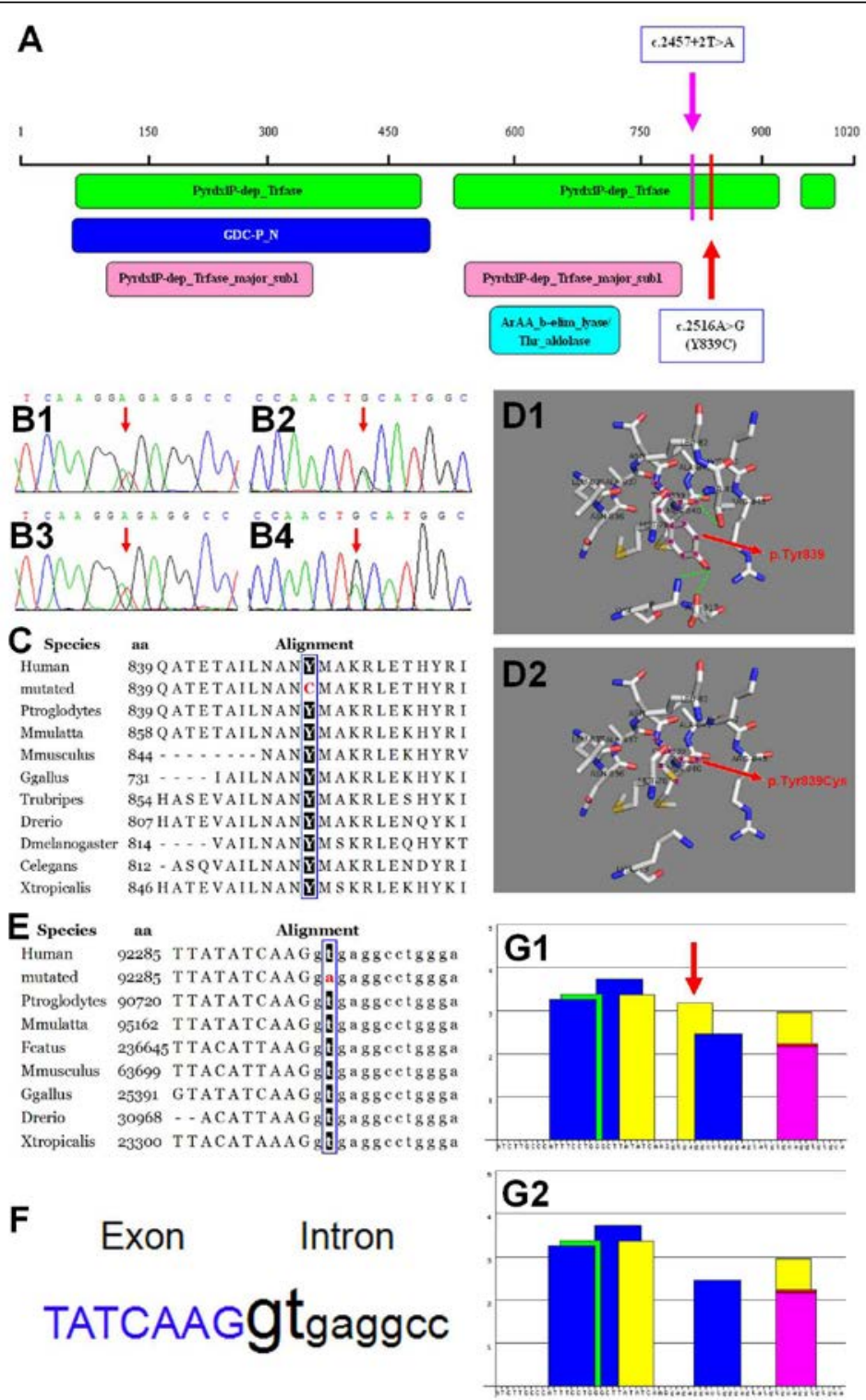

(A) Graphical view of protein domain and structure of GLDC. The green, blue, pink and cyan boxes respectively represent the domains of pyridoxal phosphate-dependent transferase (IPR015424), glycine cleavage system P-protein, N-terminal (IPR020580), pyridoxal phosphate-dependent transferase, major region, subdomain 1 (IPR015421) and aromatic amino acid beta-eliminating lyase / threonine aldolase (IPR001597). The point mutations of c.2457+2T>A and c.2516A>G(Y839C) in GLDC are marked as purple and red lines respectively.

(B) Sequence chromatograms of the GLDC mutations. B1 and B2 respectively represent the mutated sequences of c. $2457+2 \mathrm{~T}>\mathrm{A}$ and c.2516A >G. in the boy, which inherit from his father (B3) and mother (B4). The mutation site is indicated (red arrows).

(C) BLAST comparison of the sequence around amino acids 839 in orthologs of GLDC among species. The block and characters indicate the amino acid highly conserved among species in which p.Tyr839Cys was identified.

(D1 and D2) Computer simulation of the amino acids and structures around position 839 in GLDC. The cartoon structures of wild type (D1) and mutant type (D2) GLDC show that mutagenesis replacing tyrosine with cysteine at position 839 in the pyridoxal phosphate-dependent transferase domain of the glycine dehydrogenase. In D1, the dotted green lines indicate steric interactions around tyrosine at position 839, while D2 indicates losing of the steric interactions and conformational change of the domain introduced as a result of substitution.

(E) BLAST comparison of the sequence around nucleotide c. $2457+2$ in orthologs of GLDC among species. The block and characters indicate the nucleotide highly conserved among species in which c.2457+2T $>$ A was identified.

(F) Sequence diagram of the c.2457+2 indicates that the mutation was near the exon-intron boundary.

(G1 and G2) Computational prediction of the effect of c.2457+2T>A change on exon-intron splicing enhancer composition. G1: The $\mathrm{T}$ at position +2 of the 20th intron of the GLDC gene includes an ESE. G2: Note that the T to A substitution loses the exon-intron splicing enhancer in the 20th intron recognized by the SRSF6 protein. The red arrow indicates the mutation position. 
and excess glycine could account for seizures. Mutations in the GLDC gene are responsible for $70 \%-75 \%$ of NKH cases, whereas mutations in the AMT and GCSH genes only account for $20 \%$ and $<1 \%$ of NKH cases respectively. ${ }^{3}$ Recent research revealed that a few patients with enzymatic deficiency don't have an identified mutation in $G L D C$, either AMT or GSH. These cases are due to deficiency in the synthesis of cofactor lipoic acid, ${ }^{10,11}$ or disruption of the glycine transport system which is mediated by two sodiumdependent carriers, mainly GLYT1 and GLYT2. ${ }^{12}$ For the primary disease-causing gene, there are 214 reported pathogenic variants in GLDC up to now, and no mutational hotspots was found. Missense mutations account for more than 70\% of the variants, the others being larger-scale deletions, small-scale insertions/deletions, and mutations that may affect splicing (The Human Gene Mutation Database 2016). ${ }^{13}$

The c. $2457+2 \mathrm{~T}>\mathrm{A}$ mutation in exon 20 , is predicted to disrupt the splice site and affect alternative splicing of mRNA. The c.2516A $>$ G missense mutation in exon 21 leads to the substitution of tyrosine with cysteine at 839 th position of the $\mathrm{P}$ protein. These two mutations have not been reported in the Human Gene Mutation Database (HGMD), dbSNP database and PubMed. A suite of bioinformatics programmes have indicated that these two mutations are highly likely to be pathogenic. As functional analysis were not done, further studies and patients are needed.

The identification of the two novel mutations of GLDC gene in the boy suggests that nonketotic hyperglycinemia is the most probable cause of the clinical manifestations of the boy. The two novel mutations in the GLDC gene, which are associated with absent enzymatic activity and elevated glycine levels, also correlated with the severe phenotype in this boy. In addition, the verification of the two novel mutations in the parents confirms their carrier status. With the availability of the mutations report, prenatal diagnosis could be offered for future pregnancies.

\section{REFERENCES}

1. Madu AE, Oliver L. Non-ketotic hyperglycinaemia: case report and review of medical literature. J Matern Fetal Neonatal Med 2013;26(5):537-9.

2. Beyoğlu D, Idle JR. The glycine deportation system and its pharmacological consequences. Pharmacol Ther 2012;135(2):151-67.

3. Van Hove J, Coughlin C II, Scharer G. Glycine Encephalopathy. In: Pagon RA, Adam MP, Ardinger HH, et al. Gene Reviews ${ }^{\circledR}$ [Internet]. Seattle(WA): University of Washington, Seattle; 1993-2017.

4. Hennermann JB, Berger JM, Grieben U, et al. Predicition of long-term outcome in glycine encephalopathy: a clinical survey. J Inherit Metab Dis 2012;35:253-61.

5. Wang W, Wu Z, Dai Z, et al. Glycine metabolism in animals and humans: implications for nutrition and health. Amino Acids 2013;45(3):463-77.

6. Coughlin CR 2nd, Swanson MA, Kronquist K, et al. The genetic basis of classic nonketotic hyperglycinemia due to mutations in GLDC and AMT. Genet Med 2017;19(1):104-11.

7. Narisawa A, Komatsuzaki S, Kikuchi A, et al. Mutations in genes encoding the glycine cleavage system predispose to neural tube defects in mice and humans. Hum Mol Genet 20121;21(7):1496-503.

8. Bjoraker KJ, Swanson MA, Coughlin CR 2nd, et al. Neurodevelopmental Outcome and Treatment Efficacy of Benzoate and Dextromethorphan in Siblings with Attenuated Nonketotic Hyperglycinemia. J Pediatr 2016;170:234-9.

9. Love JM, Prosser D, Love DR, et al. A novel glycine decarboxylase gene mutation in an Indian family with nonketotic hyperglycinemia. J Child Neurol 2014;29(1):122-7.

10. Baker PR 2nd, Friederich MW, Swanson MA, et al. Variant non ketotic hyperglycinemia is caused by mutations in LIAS, BOLA3 and the novel gene GLRX5. Brain 2014;137(Pt 2):366-79.

11. Mayr JA, Zimmermann FA, Fauth C, et al. Lipoic acid synthetase deficiency causes neonatal-onset epilepsy, defective mitochondrial energy metabolism, and glycine elevation. Am J Hum Genet 2011;89(6):792-7.

12. Alfadhel M, Nashabat M, Qahtani HA, et al. Mutation in SLC6A9 encoding a glycine transporter causes a novel form of non-ketotic hyperglycinemia in humans. Hum Genet 2016;135(11):1263-8.

13. The Human Gene Mutation Database at the Institute of Medical Genetics is Cardiff. 2016. Professional 2016.4. [Accessed on: march 29, 2017] Available at: http: / / www. hgmd.cf.ac.uk/ac/index.php. 